\title{
Chronische Zystitis
}

Eine effektive Behandlung der chronischen (interstitiellen) Zystitis ist aufgrund der Belastung der Patienten dringend geboten. Die Therapie zur Wiederherstellung des geschädigten Urothels ist nun auch mit einem speziellen Adapter möglich, was von Betroffenen gut angenommen wird, berichtet Dr. Alexander Dürer im Gespräch mit dem Journal für Urologie und Urogynäkologie.

Herr Dr. Dürer, eine chronische Zystitis kann für viele Betroffene sehr belastend sein. Wie sieht die Symptomatik in der Praxis aus, was berichten die Patientinnen?

Dürer: Chronische Entzündungen der Blase bedeuten für viele Patientinnen und Patienten auch chronisches Leid. Die Betroffenen berichten diesbezüglich vor allem über die stark verkürzten Miktionsintervalle und den quälenden Harndrang, der teilweise mit Unterbauchschmerzen gepaart ist. Diese Symptome liegen häufig seit Jahren vor. Sehr oft wird die Diagnose Harnwegsinfekt gestellt und dagegen immer wieder Antibiotika verschriebenen, die dann aber ohne Wirkung bleiben. Dies ist für die Patienten natürlich überaus belastend. Ich würde daher speziell bei häufigen dysurischen Beschwerden und unklaren Unterbauchschmerzen empfehlen, die Patienten einer urologischen Abklärung zuzuführen und eine interstitielle Zystitis zu erwägen.

Ialuril ${ }^{\circledast}$ mit den 3 Komponenten Hyaluronsäure (1,6\%), Chondroitinsulfat (2\%) und Calciumchlorid $(0,87 \%)$ wird zur Wiederherstellung der GAG-Schutzschicht des UrothelBlasengewebes eingesetzt. Für diese Therapie ist nun ein neuer Adapter (laluadapter ${ }^{\circledast}$ ) verfügbar. Für welche Patientengruppen ist er besonders geeignet?

Dürer: Eigentlich gibt es hier keine Einschränkungen, der Adapter ist grundsätzlich für alle Patientengruppen - Ältere und Jüngere gleichermaßen geeignet. Ich würde sagen, die einzige Ausnahme stellen vielleicht Patientinnen mit ausgeprägter Adipositas dar, bei denen eine Applikation des Wirkstoffs nicht so leicht ist; in diesen Fällen kann es besser sein, doch auf den Einmalkatheter zurückzugreifen.

Was ist vor der ersten Anwendung des Adapters zu beachten? Welche Informationen sind für Patienten besonders wichtig?

Dürer: Die Patientin beziehungsweise der Patient ist darüber aufzuklären, dass sie oder er nun einen Monat lang eine wöchentliche Therapie und anschließend eine Erhaltungstherapie erhält, vor deren Instillation die Blase zu entleeren ist. Der nächste Toilettengang ist so lange wie möglich hinauszuschieben - je länger, umso besser, aber zumindest 30 Minuten lang sollte man nach der Behandlung nicht urinieren.

Welche Vorteile gegenüber dem Katheter haben Sie bei der Verwendung des Adapters festgestellt?

Dürer: Der Katheter wird in die Harnblase eingeführt, der Adapter wird hingegen nur bei der äußeren Harnröhrenöffnung mit leichtem Druck angesetzt, um einen guten Abschluss zu gewährleisten; danach kann der Wirkstoff bereits appliziert werden. Für die meisten Patienten ist der Adapter eindeutig angenehmer als der Katheter. Ich habe beobachtet, dass für viele Patienten schon allein die Vorstellung, keinen Katheter eingesetzt zu bekommen, eine entspannende Wirkung zeigt. Man muss aber auch sagen, dass es einen geringen Patientenanteil gibt, der nach wie vor den Katheter vorzieht, wahrscheinlich deswegen, weil bei der Adapter-Therapie schon ein gewisser Druck auf den Meatus ausgeübt werden muss. Für mich als applizierenden Arzt ist damit ein kleiner Zeitvorteil verbunden, da ich keinen Katheter extra herrichten muss. Ich schließe den Adapter an die Spritze an, und kann gleich die Behandlung durchführen.

Wie sieht es bei der Restharnentnahme aus?

Dürer: Wenn ich den Restharn noch kontrollieren will, dann muss ich das sonographisch oder mittels Einmalkatheter machen, nicht mit dem Adapter.

Sie benutzen den Adapter seit einigen Monaten, wie würden Sie die Erfahrungen zusammenfassen? Dürer: Ich habe im Rahmen einer Testphase beide Methoden bei meinen Patienten ausprobiert und dann nachgefragt, welche die angenehmere Variante ist. Und hier haben vier von fünf Patientinnen den Adapter durchwegs als positiv bewertet und diese Methode dem Katheter vorgezogen. Jede fünfte Patientin wollte allerdings beim Katheter bleiben.

\section{Vielen Dank für das Gespräch!}

Das Gespräch führte Dr. Lydia UngerHunt.

J. Urol. Urogynäkol. AT 2019 · 26:77 https://doi.org/10.1007/s41972-0190075-1

(c) Springer-Verlag GmbH Austria, ein Teil von Springer Nature 2019

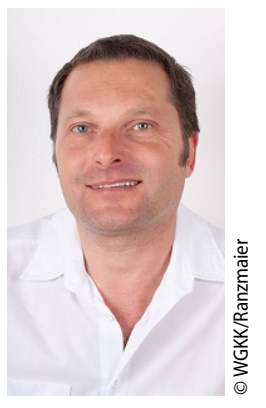

Dr. Alexander Dürer 